\title{
Interações estabelecidas pelas Universidades Brasileiras Públicas e Privadas*
}

\author{
Matheus Schmidt ${ }^{\dagger}$ \\ Janaina Ruffoni $\ddagger$
}

\begin{abstract}
Resumo
As universidades são reconhecidas como produtoras e difusoras de conhecimento cientifico e tecnológico, e podem influenciar na geração e difusão de inovações. Para a realidade brasileira, a literatura aponta um volume relativamente baixo de interações entre universidades e organizações, considerando a imaturidade do Sistema Nacional de Inovação (SNI) do país. Além disso, entendese que as universidades públicas, em comparação com as privadas, são instituições com maior presença no cenário de interações, pela trajetória histórica de constituição e desenvolvimento de mais longo prazo no sistema educacional e de ciência e tecnologia brasileiro, bem como pelo fato de usufruírem de mais recursos, tanto humanos quanto financeiros para a geração e transmissão de conhecimentos. Neste contexto, entende-se ser relevante compreender as interações estabelecidas de acordo com a natureza jurídica das universidades. Para tanto, foi realizado um estudo exploratório que utilizou dados secundários do censo do Diretório de Grupos de Pesquisa (DGP) do CNPq e do censo de educação superior do INEP para o ano de 2014. Os resultados indicaram que as universidades públicas possuem um número maior de registros de grupos de pesquisa, grupos de pesquisa interativos, doutores, mestres e outros recursos em relação às universidades privadas, sendo as principais áreas de conhecimento dos grupos interativos: ciência da saúde, ciências humanas e ciências exatas e da terra. Já os grupos interativos das universidades privadas são principalmente das áreas de ciências sociais aplicadas, engenharias e ciências biológicas. Outro resultado interessante, e surpreendente, foi o cálculo do grau de interação que apontou ter sido semelhante para os dois grupos de universidades analisados. Isso exige um olhar mais acurado e inclusivo do papel das universidades privadas no cenário da interação universidades-organizações no Brasil.
\end{abstract}

Palavras-chave: Interação Universidade e Organizações, Universidades Públicas e Privadas, Grupos de Pesquisa Interativos, Sistema Nacional de Inovação Brasileiro.

JEL: O33

*Esse trabalho é resultado da dissertação de mestrado de Schmidt, M. (2017).

${ }^{\dagger}$ Mestre em Economia pelo Pós-Graduação em Economia (PPGE) da Universidade do Vale do Rio dos Sinos (UNISINOS).

†Professora do Programa de Pós-Graduação em Economia (PPGE) da Universidade do Vale do Rio dos Sinos (UNISINOS). 


\section{Introdução}

A partir dos estudos de Schumpeter $(1982,1961)$, o desenvolvimento econômico pode ser entendido por rupturas no sistema capitalista resultantes de inovações paradigmáticas que reajustam o funcionamento do sistema. A compreensão do processo inovativo como variável endógena ao sistema econômico foi um passo fundamental para o surgimento da denominada Teoria Neo-Schumpeteriana e, desde então, a inovação começou a receber espaço na literatura econômica como elemento essencial para o entendimento do progresso técnico.

A partir da compreensão de que o processo de geração de inovações é influenciado pela existência de instituições estimuladoras da mudança (governos, instituições de pesquisa, universidades, etc.) e que também ocorre por meio da interação entre estas diferentes instituições, surge, na década de 1980, o conceito de Sistema Nacional de Inovação (SNI). Autores como Freeman (1995), Lundvall (1992), Nelson (1993), todos neoschumpeterianos, definem SNI como uma rede de instituições dos setores público e privado, em que as atividades e interações iniciam, modificam e difundem novas tecnologias, incluindo todas as partes e aspectos da estrutura econômica e quadro institucional que afeta a aprendizagem e pesquisa, ou conjunto de instituições cujas interações determinam o desempenho inovador das empresas nacionais (ALBUQUERQUE, 1996).

Frente a esse contexto, o ator 'Universidade', mostra-se fundamental no SNI também para geração e difusão de inovações, além das suas funções de pesquisa e ensino. Neste sentido, a interação Universidade e setor produtivo (aqui denominado de UniversidadeOrganizações) tem aparecido de forma crescente nas discussões acadêmicas.

No cenário brasileiro, o caráter tardio do desenvolvimento do sistema de ensino superior público, a reestruturação da infraestrutura acadêmica na década de 1980, as definições de políticas públicas destinadas ao ensino e à pesquisa e o posterior surgimento de um sistema paralelo de ensino superior privado tornaram o contexto das universidades e suas relações repleto de especificidades (DURHAM, 2003).

Ainda que Instituições de Ensino Superior (IES) privadas absorvam a grande demanda de qualificação formal do país (Brasil) por matriculas em graduação, a maior parte da pesquisa produzida, principalmente básica, é fruto do investimento público em universidades públicas, evidenciando ainda mais as diferenças entre as instituições (SGUISSARDI, 2005).

Considerando esse contexto de diferenças entre universidades públicas e privadas, este artigo tem como objetivo compreender as características das interações estabelecidas por universidades públicas e privadas a partir de uma análise empírica e descritiva de dados secundários para o ano de 2014.

Econômica-Niterói, v. 20, n. 2, p. 33-58. Dezembro, 2018 
Para tanto, o artigo está estruturado em mais quatro seções. A próxima apresenta um breve panorama a respeito dos elementos relevantes para a discussão relativa à interação universidade-organizações no Brasil. Na terceira estão presentes os procedimentos metodológicos. Na quarta são apresentados e analisados os resultados. E, por fim, na quinta seção são desenvolvidas as conclusões do estudo.

\section{Universidades, Sistema Nacional de Inovação e Interações no Brasil}

O Brasil iniciou a criação de universidades tardiamente, se comparado com o grupo de países desenvolvidos (DURHAM, 2003). Embora a literatura aponte o início da pesquisa cientifica no Brasil na década de 1920, a primeira Universidade criada no país foi a USP no ano de 1934 incorporando a antiga Escola Politécnica (inaugurada em 1894), a Faculdade de Farmácia (1898), a Faculdade de Medicina e Cirurgia (1912), o Instituto de Veterinária (1919), o Instituto Biológico (1924), entre outras instituições (SCHARTZMAN, 1979).

A literatura aponta que, além da lenta formação de instituições pertencentes ao Sistema Nacional de Inovação (SNI), o Brasil realizou um processo de industrialização tardio, repercutindo, em geral, em uma tímida demanda pelo desenvolvimento de inovações nacionais. Para Suzigan e Albuquerque (2008, p. 24) "alguns aspectos da especialização cientifica brasileira atual podem ser compreendidos pelos elementos apontados pelas trajetórias das ciências da saúde, ciências agrárias, mineração, engenharia de materiais e metalúrgica, e engenharia aeronáutica". Essa contextualização auxilia na compreensão de uma das questões abordadas pela literatura quando trata do SNI Brasileiro, seus limites e sua baixa interação entre os agentes que o compõe.

Para Suzigan e Albuquerque (2006), o Brasil ainda não possui um sistema de inovação completo. O país faz parte de um grupo de nações que ainda não atingiu tal nível de maturidade. Entre os países sinalizados neste grupo ainda há a Índia, África do Sul, México, entre outros.

Compreender que no Brasil o SNI teve um começo tardio, limitado e problemático é fundamental para instigar estudos a respeito das suas características atuais com vistas a qualificar tal sistema. Com base na teoria neoschumpeteriana, um SNI bem estruturado, maduro e dinâmico é um importante alicerce para o desenvolvimento geral de uma nação. Ano surgiment Instituições de Ensino Superior (IES) e de Pesquisa no Brasil é considerado um componente importante para compreender os limites do SNI atual (SUZIGAN; ALBUQUERQUE, 2008).

As universidades, juntamente com as empresas, devem se envolver na produção de conhecimento de fronteira, em especial de conhecimento de potencial aplicabilidade para o setor produtivo, auxiliando assim no desenvolvimento do SNI. Conforme as palavras da Rapini (RAPINI, 2007, p. 213):

As contribuições das universidades para o processo inovativo nas firmas

Econômica-Niterói, v. 20, n. 2, p. 33-58. Dezembro, 2018 


\begin{abstract}
são sintetizadas como: fonte de conhecimento de caráter mais geral necessários para as atividades de pesquisa básica (Nelson, 1990); fonte de conhecimento especializado relacionado a área tecnológica da firma (Klevoricfonte de s de lidar com problemas associados ao processo inovativo nas firmas (Rosenberg e Nelson 1994); Pavitt (1988); criação de novos e de técnicas cientifica (Rosenberg 1992); criação de firmas nascentes (Spin-offs) por pessoal acadêmico (Stankiewicz, 994; Etzkowitz, 1999).
\end{abstract}

Segundo Rapini e Righi (2007), no contexto brasileiro, o relacionamento entre universidades e empresas ocorria através de um fluxo unidirecional que tinha sua origem nas universidades, corroborando com o pensamento de Schartzman (1986), quando afirma que o sistema público de pesquisa, ao contrário do senso comum, estaria cada vez mais engajado na pesquisa aplicada, apresentando uma predisposição para a cooperação com o setor produtivo.

Ao analisar os dados dos Censos do Diretório de Grupos de Pesquisa (DGP) do Conselho Nacional de Desenvolvimento Científico e Tecnológico (CNPq) de 2002 a 2010, Silva et al. (2015) apontam para a intensificação do número de interações estabelecidas pelas universidades brasileiras. O estudo apontou que no nível nacional, o percentual de grupos de pesquisa que declaram ter interação com outras organizações não apenas cresceu em números absolutos, como também aumentou sua representatividade em relação ao total de grupos de pesquisa registrados no país.

Puffal, Ruffoni e Schaeffer (2012), que analisaram dados de duas pesquisas survey nacionais realizadas entre 2008 e $2009^{1}$ com empresas que interagiam com universidades e com grupos de pesquisa que interagiam com empresas no Brasil, afirmaram, em relação aos interesses dos atores envolvidos nas interações, que existem interesses distintos entre esses, com predomínio de ações de curto prazo. Tal característica corrobora com o perfil de baixo nível inovativo das empresas brasileiras. Já em relação às razões que motivavam universidades a buscar a interação com empresas, essas eram, em sua essência, acadêmicas. Na ótica das empresas, as motivações citadas estavam diretamente relacionadas à falta de recursos internos, ou a ampliação da possibilidade de desenvolver tecnologia com menor investimento, diminuindo os riscos assumidos no processo.

Em outro trabalho semelhante, mas com um recorte regional para o Rio Grande do Sul, Schaeffer, Ruffoni e Puffal (2015) apresentaram os resultados de uma pesquisa survey que investigou interacoes na area de conhecimento das ciencias da computacao no RS em 2013. As principais razoes e beneficios das interações analisadas para as universidades classificaram-se como intelectuais, enquanto que para as empresas as razoes são proativas e os beneficios são aqueles focados em inovacao. Em relacao as dificuldades do estabelecimento das interações, os dois grupos de respondentes (líderes de grupos de pesquisa e empresários) destacaram questões de prioridade e burocracia. Os autores apontaram

\footnotetext{
${ }^{1}$ Denominada de Brazil Survey ou BR Survey. Foi executada por uma rede nacional de pesquisa coordenada pelos Professores Wilson Suzigan, da UNICAMP, e Eduardo da Motta e Albuquerque, da UFMG.
}

Econômica-Niterói, v. 20, n. 2, p. 33-58. Dezembro, 2018 
para uma diferença em relação à burocracia no que diz respeito à natureza jurídica das universidades, uma vez que a percepção da burocracia no processo de interação foi superior na universidade publica em relação à universidade privada. Entendeu-se, portanto, no estudo realizado, que os mecanismos adotados pelas universidades publicas para regular as interacoes parecem ser mais complexos.

Pinho (2011) aponta para uma grande importância das universidades brasileiras para a geração de novos projetos e no auxilio à conclusão de projetos antigos, já iniciados nas empresas, mas possui uma participação menos destacada nas indústrias de alta tecnologia e nos serviços de informação e comunicação.

Para Stal et al. (2016) a literatura brasileira sobre interação entre universidades e empresas ainda discute questões já superadas nos EUA e na Europa. Existem diversos artigos que discutem os prós e contras da interação sob o ponto de vista da academia, o modelo mais adequado para transferência de tecnologia, direitos de propriedade intelectual (propriedade individual e privilégio do professor ou propriedade institucional), sistemas nacionais e regionais de inovação, entre outros. Porém, o debate a respeito da necessidade da interação, já se mostra superado, embora não seja observada unanimidade da universidade nesta questão.

Países em desenvolvimento caracterizam-se pela pouca ou inexistente interação entre as diversas empresas e universidades na solidificação de uma trajetória de desenvolvimento apoiada em inovação. Se tratando da interação entre universidades e o setor produtivo, a falta de estratégias de concorrência baseadas na geração interna de conhecimento por parte das empresas acaba resultando em desinteresse por relacionamentos próximos a universidades. Embora existam trabalhos publicados na área da tecnologia e inovação que comprovam o papel crucial que a interação entre produção cientifica e o setor empresarial assume dentro da economia da tecnologia (RAPINI; RIGHI, 2007).

A empresa brasileira, ainda que interessada em incorporar conhecimento em seu produto, parece não confiar totalmente na universidade local, ou por ignorar a capacidade cientifica do país, ou por não incluir a inovação entre as próprias prioridades (CHAIMOVICH, 1999). Também há alguns estudos que buscam relacionar o nível de capacidade absortiva da firma e o estabelecimento de interações com universidades (ROSA; RUFFONI, 2013). Isso, pois é de entendimento que para buscar a interação é necessário ter condições de absorver o conhecimento do agente parceiro. Não basta ter uma boa infraestrutura e oferta de ciência e tecnologia em um país para que ocorram interações entre os atores das esferas de ciência e de tecnologia. É necessário mais do que isso, é preciso que os atores tenham capacidade de absorver os conhecimentos externos, que são de domínio de outros agentes.

A respeito da capacidade de absorção, a pesquisa de Rosa e Ruffoni (2013), realizada em firmas que interagiam com grupos de pesquisa das áreas de Engenharia Mecânica, de Materiais e Metalúrgica das Universidades do Rio Grande do Sul, identificou que os tipos de interação que dizem respeito ao desenvolvimento conjunto de conhecimento, tais 
como "contratação de pesquisa científica sem considerações de uso imediato nos resultados" e "atividades de engenharia não-rotineira, inclusive o desenvolvimento de protótipo, cabeça de série ou planta-piloto, desenvolvida para a empresa pela Universidade", são mais efetuados por firmas que possuem capacidade absortiva alta. Conforme as autoras, tais resultados confirmam o que já foi destacado por Meyer-Krahmer e Schmoch (1998) ao estabelecer a capacidade absortiva como um fator determinante para a interação de empresas com universidades.

A discussão a respeito das interações estabelecidas pelas universidades brasileiras, por meio de seus grupos de pesquisa, também exige um olhar crítico a respeito do papel que a Universidade efetivamente assume no SNI de países periféricos. Os autores Brundenius, Lundvall e Sutz (2009) afirmam que o papel da Universidade nos países em desenvolvimento deve ser o de uma 'Developmental University', pois há peculiaridades nestas regiões que devem ser respeitadas quando da análise do papel deste importante ator no SNI, tais como: fontes de recursos escassas, demanda por conhecimento tecnológico e científico relativamente fraca (devido ao restrito cenário de inovação do setor produtivo), tendências de privatização do ensino superior (o que pode ameaçar a qualidade das suas atividades), entre outras. Os autores defendem a perspectiva da 'Developmental University' afirmando que

\footnotetext{
"We have indicated that we see the diversified 'developmental university' system as a possible response to the contradictory demands confronting universities in the current era. Such systems combine a capacity to respond in the short term to the needs of users with some degree of autonomy and long-turn commitment. They also aim at promoting innovation in such a way that it can be combined with social and global equality and justice" (BRUNDENIUS; LUNDVALL; SUTZ, 2009, p. $331)$.
}

Portanto, estudos na temática da interação entre universidades e organizações são fundamentais para melhor compreender esse fenômeno na economia brasileira. Entende-se que isso contribui para uma análise da dinâmica existente entre os atores do SNI Brasileiro. Diversos são os estudos já realizados a respeito do tema no país ${ }^{2}$. Entretanto, não foi identificado um que abordasse as características das interações considerando a natureza jurídica das universidades, sendo, portanto, o objetivo do estudo aqui apresentado. Assim, entende-se que este trabalho contribui para as pesquisas relativas ao entendimento das interações estabelecidas entre universidades e organizações do setor produtivo e como isso pode influenciar na dinâmica do SNI Brasileiro.

\footnotetext{
${ }^{2}$ Pelo menos duas publicações reúnem estudos a respeito desta discussão: o número especial da 'Revista de Economia' da Universidade Federal do Paraná (UFPR), vol. 37 (número especial), ano 35, 2011, e o livro organizado por Wilson Suzigan, Eduardo da Motta e Albuquerque e Silvio Antônio Ferraz Cario, intitulado 'Em busca da inovação: interação universidade-empresa no Brasil', de 2011.
}

Econômica-Niterói, v. 20, n. 2, p. 33-58. Dezembro, 2018 


\section{Procedimentos metodológicos}

O estudo foi guiado com base em um método exploratório, com corte transversal, com o uso de dados secundários disponíveis no Diretório dos Grupos de Pesquisa (DGP) do CNPq e do Censo de Educação Superior do Instituto Nacional de Estudos e Pesquisas Educacionais Anísio Teixeira (INEP), ambos para o ano de 2014.

O diretório dos Grupos de Pesquisa (DGP) do CNPq é o inventário dos grupos de pesquisa cientifica e tecnológica em atividades no Brasil. As informações nele contidas referem-se aos recursos humanos que constituem os grupos de pesquisa, (estudantes e pesquisadores) assim como suas principais atividades de produção cientifica, especialidades dos conhecimentos, setores de aplicação envolvidos, produção cientifica e tecnológica e parcerias estabelecidas com instituições e as linhas de pesquisa em andamento, gerando assim um perfil geral de todas as atividades cientifico-tecnológica no Brasil. No DGP são reunidas informações de diversas instituições, entre elas universidades estaduais, particulares, institutos de pesquisa, instituições públicas tecnológicas, laboratórios $\mathrm{P} \& \mathrm{D}$ do estado e de empresas privadas, Organizações não governamentais (ONG) envolvidas em pesquisa cientifica e tecnológicas. Os grupos de pesquisa registrados estão localizados, em sua grande maioria, em universidades e institutos de pesquisa. Já o censo da Educação superior elaborado pelo Instituto Nacional de Estudos e Pesquisas Educacionais Anísio Teixeira (INEP) é realizado anualmente e possui como objetivo oferecer a comunidade acadêmica informações detalhadas sobre a situação do setor no contexto brasileiro. $O$ censo reúne informações sobre as instituições de ensino superior do país coletados através de questionário enviado as instituições e importação de dados do sistema e-mec ${ }^{3}$.

Para o ano de 2014, identificou-se um total de 35.424 grupos de pesquisa cadastrados, pertencentes a 492 organizações de diferentes tipos, cadastradas no DGP. Entre elas, estão institutos de pesquisa, laboratórios de $\mathrm{P} \& \mathrm{D}$, organizações não governamentais $(\mathrm{ONG})$, faculdades, centros tecnológicos, fundações e universidades.

$\mathrm{Na}$ base de dados existem as chamadas instituições interativas, ou seja, aquelas que estabelecem interação com uma ou mais empresas; o total destas instituições é de 422, as quais têm ao todo 9.348 grupos que registraram uma ou mais interações com empresas.

A pesquisa realizada concentrou-se em um tipo de organização existente no DGP/CNPq: as universidades, as quais foram classificadas conforme o credenciamento no Ministério da Educação (MEC). A unidade de análise da pesquisa foram os grupos de pequisa. Dessa forma, foram selecionados os grupos de pesquisa de todas as universidades listadas na base de dados do censo do DGP de 2014, classificados em interativos ou não, pertencentes a universidades públicas ou privadas. O foco de análise do estudo centrou-

\footnotetext{
${ }^{3} \mathrm{O}$ e-MEC é um sistema eletrônico de acompanhamento dos processos que regulam a educação superior no Brasil pertencente ao Ministério da Educação. Todos os pedidos de credenciamento e recredenciamento de instituições de educação superior e de autorização, renovação e reconhecimento de cursos, são realizados pelo sistema e-MEC.
} 
se nos grupos interativos, pertencente aos diferentes tipos de universidades referentes à dimensão nacional.

Considerando as instituições selecionadas, efetuou-se um corte metodológico selecionando variáveis de estrutura (institucional e de recursos humanos) e de resultado definidas a partir de apropriações destes conceitos na base de dados, conforme já realizado em pesquisas anteriores (SILVA, 2016).

Além das variáveis fornecidas pelo banco de dados do DGP, foram utilizados outros indicadores a fim de auxiliar na discussão a respeito da diferença entre grupos de universidades. Foram considerados indicadores de interação - grau de interação e diversidade de interação institucional - calculados com base nas informações do banco de dados e financeiros ${ }^{4}$ (Receitas e despesas institucionais) registradas no censo da educação superior do INEP (Instituto Nacional de Estudos e Pesquisas Educacionais Anísio Teixeira) para o ano de 2014 (SILVA, 2016). Conforme demonstra o quadro 1 a seguir.

A tabulação dos dados foi construída a partir do arquivo $\mathrm{XML}^{5}$ da base censitária de 2014 do DGP, juntamente com as demais informações coletadas do INEP, resultando em uma grande matriz de dados, onde os grupos interativos foram estruturados conforme a instituição pertencente, que por sua vez foi classificada de acordo com a organização acadêmica (Universitária ou Não Universitária) descartando as instituições não universitárias, e organizando as instituições restantes conforme sua natureza Jurídica (Pública ou Privada), formando assim as linhas da matriz.

As variáveis foram dispostas nas colunas conforme as dimensões apresentadas. Como muitas informações estão disponíveis somente para os grupos de pesquisa e não para instituições, foi calculada a soma dos grupos pertencentes a cada universidade, gerando assim o resultado especifico da instituição, ou grupo de instituições (publico ou privado) conforme exemplo elaborado na figura 1.

Apenas as variáveis GII e DII foram calculadas de forma distinta. O grau da interação é a razão entre o total de grupos e total de grupos interativos, permitindo que o cálculo fosse realizado igualmente para universidades e grupos de universidades. Já a Diversidade de relacionamento Institucional é a proporção do registro dos relacionamentos de cada instituição, dentre o total de 14 diferentes tipos registrados no DGP. O cálculo da DII foi realizado para cada grupo interativo, e para o cálculo do total da universidade e grupo de universidade foi utilizada a média ponderada.

\footnotetext{
${ }^{4}$ Há que se ter cautela na análise destes dados, pois é necessário considerar questões contábeis das Universidades informadas no censo de Educação Superior do INEP em 2014. Talvez possa haver subdimensionamento de alguns valores das Universidades Públicas. Recomenda-se maior aprofundamento do entendimento destes dados para análises futuras.

${ }^{5}$ O Plano Tabular foi descontinuado após o Censo de 2010. Contudo, o CNPq disponibiliza o XML - http://lattes.cnpq.br/web/dgp/censos2 - para que seja realizada a consulta à base dados de cada um dos censos realizados. Até a data de conclusão desta pesquisa, o arquivo XML do censo de 2016 do DGP/CNPq não havia sido disponibilizado.
}

Econômica-Niterói, v. 20, n. 2, p. 33-58. Dezembro, 2018 
Tabela 1 - Variáveis utilizadas no estudo

\begin{tabular}{|c|c|}
\hline \multicolumn{2}{|r|}{1 - Variáveis de Estrutura } \\
\hline QTDGP & Quantidade de grupos de pesquisa \\
\hline QTDPREL & $\begin{array}{l}\text { Quantidade de grupos de pesquisa interativos (com um ou mais } \\
\text { relacionamentos) }\end{array}$ \\
\hline TOTALREL & Total de relacionamentos \\
\hline QTDEMPR & Quantidade de instituições parceiras \\
\hline TOTPESQ & Total de pesquisadores dos grupos interativos \\
\hline TOTEST & Total de estudantes dos grupos interativos \\
\hline LPESQ & Linhas de pesquisa \\
\hline \multicolumn{2}{|r|}{2 - Estruturas de Recursos Humanos } \\
\hline PESDOUT & Pesquisadores doutores \\
\hline PESQMEST & Pesquisadores mestres \\
\hline PESQESPEC & Pesquisadores especialistas \\
\hline PESQGRAD & Pesquisadores graduandos \\
\hline \multicolumn{2}{|r|}{3 - Interação } \\
\hline GII & Grau de interação da instituição \\
\hline DII & Diversidade de interação da instituição \\
\hline \multicolumn{2}{|r|}{4 - Investimento e Despesas Institucionais } \\
\hline RECPROPRIA & Receita própria \\
\hline VALDESPINV & Investimento total \\
\hline VALDESPEQU & Investimento em pesquisa \\
\hline VALDESPDOC & Valor das despesas docentes \\
\hline \multicolumn{2}{|r|}{5 - Resultados dos Grupos de Pesquisa } \\
\hline TOTPUBLIC & Total de publicações \\
\hline TOTPRODTEC & Total de produção técnica \\
\hline TOTORIENT & Total de orientações \\
\hline
\end{tabular}

Fonte: Elaborado pelos autores 
Figura 1 - Exemplo de cálculo das variáveis para universidades e grupos de pesquisa

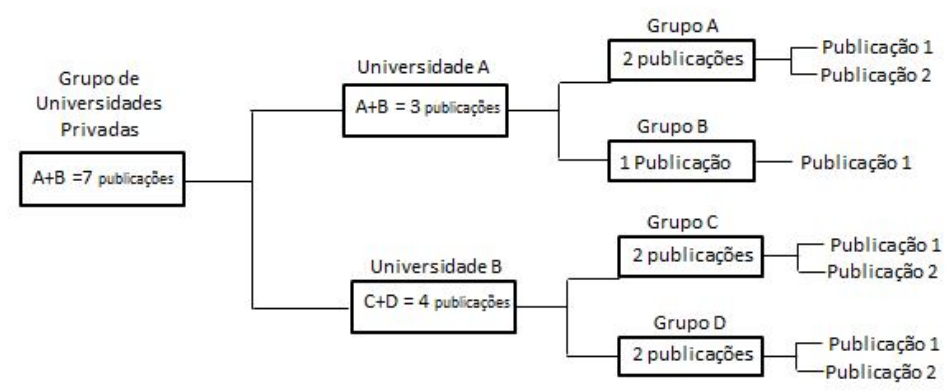

Fonte: Elaborado pelos autores.

\section{Grupos de Pesquisa Interativos das Universidades Brasileiras Pú- blicas e Privadas}

Nesta seção, analisam-se questões como o tipo de interação, grande área de conhecimento dos grupos de pesquisa, distribuição espacial das interações, entre outros. Entre as variáveis descritas estão a quantidade de grupos de pesquisa com e sem relacionamento, o total de relacionamentos, o número de empresas parceiras, a estrutura dos grupos em termos de recursos humanos (pesquisadores e estudantes). Variáveis financeiras (investimento total, investimento em pesquisa, despesas docentes). Estas variáveis são compreendidas como variáveis que podem direta ou indiretamente afetar a capacidade de interação de cada instituição. Variáveis de interação (grau da interação, diversidade da interação) resultantes dos relacionamentos registrados pelos grupos pertencentes às universidades da amostra selecionada. E por fim, variáveis financeiras de investimento e despesas institucionais (receita própria, investimento total, investimento em pesquisa e valor das despesas com docentes).

Filtrando o número de grupos conforme as áreas de conhecimento com maior número de grupos cadastrados, são destacadas as áreas de ciências da saúde e ciências humanas. A tabela 2 apresenta um panorama geral contendo o total de grupos e grupos interativos pertencentes a universidades, conforme DGP 2014.

Ao analisarmos os grupos filtrando pela natureza jurídica das universidades, obtemos resultados diferentes. Para as instituições de natureza jurídica pública, as áreas predominantes são Ciências da Saúde, Ciências Humanas e Ciências Exatas e da Terra. Já para as instituições de natureza jurídica privada, as áreas de pesquisa predominantes foram: Engenharias, Ciências Sociais Aplicadas e Ciências Biológicas. Essas informações estão na Tabela 3.

Ao avaliarmos os tipos de relacionamentos predominantes nos grupos pertencentes às

Econômica-Niterói, v. 20, n. 2, p. 33-58. Dezembro, 2018 
Tabela 2 - Grupos de pesquisa e grupos de pesquisa interativos de universidades do Brasil por área de conhecimento.

\begin{tabular}{lccc}
\hline Área Predominante & Grupos & N. de Grupos Interativos & Grupos Interativos (\%) \\
\hline Ciências da Saúde & 1421 & 130 & 18 \\
Ciências Humanas & 1236 & 129 & 18 \\
Ciências Sociais Aplicadas & 1235 & 88 & 12 \\
Ciências Exatas e da Terra & 1138 & 105 & 15 \\
Engenharias & 922 & 94 & 13 \\
Linguística, Letras e Artes & 753 & 59 & 8 \\
Outros & 1057 & 103 & 15 \\
\hline Total & 7762 & 708 & 100 \\
\hline
\end{tabular}

Fonte: Elaborado pelos autores com base em Diretório dos Grupos de Pesquisa (DGP) do CNPq, Censo de 2014

universidades, o tipo de relacionamento "Pesquisa científica sem considerações de uso imediato dos resultados" possui o maior número de grupos registrados, assim como o maior número de grupos interativos. Em seguida temos "Pesquisa científica com considerações de uso imediato dos resultados". A seguir, podemos observar na tabela 4 como esta classificação ocorre para os grupos de universidades de diferentes naturezas jurídicas (pública e privada).

Analisando os grupos pertencentes a universidades públicas, a lista de tipos de relacionamento se altera, bem como os montantes, conforme Tabela 5.

Os resultados apresentados para os dois grupos se assemelham com os resultados apresentados para o total da amostra. As diferenças ficam no terceiro tipo de relacionamento apontado nos dois grupos. Nos grupos pertencentes às universidades privadas temos "Outros tipos predominantes de relacionamento que não se enquadrem em nenhum dos anteriores." Enquanto nas universidades públicas "Fornecimento, pelo parceiro, de insumos materiais para as atividades de pesquisa do grupo sem vinculação a um projeto específico de interesse mútuo".

A região também foi considerada um critério de análise, conforme apresentado na Tabela 6 a seguir.

As regiões que apresentam o maior registro do número de grupos de pesquisa são: Sudeste (39\%), Sul (27\%) e Nordeste (17\%). Para auxiliar na análise, foi calculado o grau de interação existente em cada região. Assim, embora exista um número absoluto menor de grupos registrados, as regiões com maior grau de interação são: Centro-oeste (11\%) Nordeste (10\%) e Norte (8\%), conforme tabela 7.

Para as universidades de natureza juridica pública, os resultados apontam para a região Nordeste com grande número de registros de grupos (19\%) e um alto percentual de grupos 


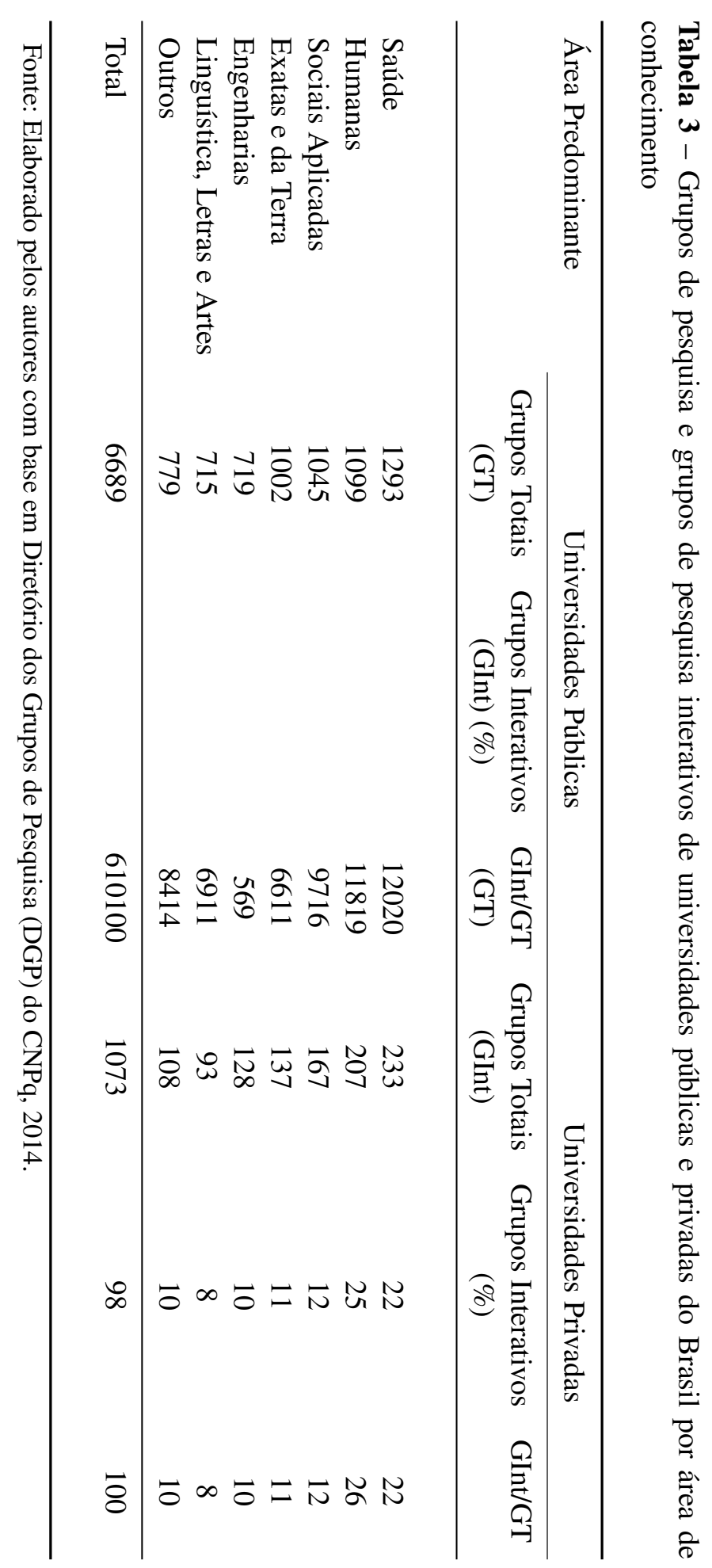

Econômica-Niterói, v. 20, n. 2, p. 33-58. Dezembro, 2018 
Tabela 4 - Grupos de pesquisa e grupos de pesquisa interativos de universidades privadas do Brasil por tipo de relacionamento

\begin{tabular}{lccc}
\hline $\begin{array}{l}\text { Tipo de relacionamento } \\
\text { predominante nas Universidades Privadas }\end{array}$ & $\begin{array}{c}\text { N. Total de } \\
\text { Grupos }\end{array}$ & $\begin{array}{c}\text { N. Total de } \\
\text { Grupos } \\
\text { Interativos }\end{array}$ & $\begin{array}{c}\text { Grupos } \\
\text { Interativos } \\
(\%)\end{array}$ \\
\hline $\begin{array}{l}\text { Pesquisa científica sem considerações de uso } \\
\text { imediato dos resultados }\end{array}$ & 843 & 79 & 81 \\
$\begin{array}{l}\text { Pesquisa científica com considerações de uso } \\
\text { imediato dos resultados }\end{array}$ & 203 & 15 & 15 \\
$\begin{array}{l}\text { Outros tipos predominantes de relacionamento } \\
\text { que não se enquadrem em nenhum dos anteriores. }\end{array}$ & 10 & 2 & 2 \\
$\begin{array}{l}\text { Transferência de tecnologia desenvolvida pelo } \\
\text { grupo para o parceiro }\end{array}$ & 6 & 0 & 0 \\
Demais Relacionamentos & 11 & 2 & 2 \\
\hline Total Geral & 1073 & 98 & 100 \\
\hline
\end{tabular}

Fonte: Elaborado pelos autores com base em Diretório dos Grupos de Pesquisa (DGP) do CNPq, 2014.

Tabela 5 - Grupos de pesquisa e grupos de pesquisa interativos de universidades públicas do Brasil por tipo de relacionamento

\begin{tabular}{lccc}
\hline $\begin{array}{l}\text { Tipo de relacionamento } \\
\text { predominante nas Universidades Públicas }\end{array}$ & $\begin{array}{c}\text { N. Total de } \\
\text { Grupos }\end{array}$ & $\begin{array}{c}\text { N. Total de } \\
\text { Grupos } \\
\text { Interativos }\end{array}$ & $\begin{array}{c}\text { Grupos } \\
\text { Interativos } \\
(\%)\end{array}$ \\
\hline $\begin{array}{l}\text { Pesquisa científica sem considerações de uso } \\
\text { imediato dos resultados }\end{array}$ & 6495 & 587 & 96 \\
$\begin{array}{l}\text { Pesquisa científica com considerações de uso } \\
\text { imediato dos resultados }\end{array}$ & 172 & 23 & 4 \\
$\begin{array}{l}\text { Fornecimento, pelo parceiro, de insumos } \\
\text { materiais para as atividades de pesquisa do } \\
\text { grupo sem vinculação a um projeto específico } \\
\text { de interesse mútuo }\end{array}$ & 17 & 0 & 0 \\
$\begin{array}{l}\text { Outros tipos predominantes de relacionamento } \\
\text { que não se enquadrem em nenhum dos anteriores }\end{array}$ & 5 & 0 & 0 \\
\hline Total Geral & 6689 & 610 & 100 \\
\hline $\begin{array}{l}\text { Fonte: Elaborado pelos autores com base em Diretório dos Grupos de Pesquisa (DGP) do CNPq, } \\
\text { 2014. }\end{array}$ & &
\end{tabular}


Tabela 6 - Distribuição dos grupos de pesquisa e grupos de pesquisa interativos por região

\begin{tabular}{lcccc}
\hline Região & $\begin{array}{c}\text { Total } \\
\text { de Grupos }\end{array}$ & $\begin{array}{c}\text { (\%) do Total } \\
\text { de Grupos }\end{array}$ & $\begin{array}{c}\text { Total de Grupos } \\
\text { Interativos }\end{array}$ & $\begin{array}{c}\text { Grau de Interação } \\
(\%)\end{array}$ \\
\hline Centro-Oeste & 650 & 8 & 69 & 11 \\
Nordeste & 1325 & 17 & 135 & 10 \\
Norte & 690 & 9 & 57 & 8 \\
Sudeste & 2992 & 39 & 281 & 9 \\
Sul & 2105 & 27 & 166 & 8 \\
\hline Total Geral & 7762 & 100 & 708 & - \\
\hline
\end{tabular}

Fonte: Elaborado pelos autores com base em Diretório dos Grupos de Pesquisa (DGP) do CNPq, Censo 2014.

com interação (10\%). A região Norte apresenta um grau de interação de $8 \%$ e o terceiro maior percentual de registros de grupos, $10 \%$. A região Centro-Oeste obteve o maior percentual do grau de interação (11\%), embora poucos registros de grupos (9\%).

Analisando os grupos pertecentes a universidades privadas, que possuem um total número menor de grupos registrados, o cenário se altera. A região Sudeste apresenta o maior percentual de grupos registrados, com 45\%, e com grau de interação de $9 \%$. A região que apresenta maior grau de interação (13\%) é a Nordeste, onde os grupos registrados somam apenas 5\% do total dos grupos. A região Sul também merece destaque por sua relação equilibrada com (39\%) de registros e (10\%) de interação.

A Tabela 8 nos mostra um panorama geral dos grupos por unidade da federação.

Em relação aos parceiros escolhidos pelos grupos de pesquisa, o Gráfico apresentado pela figura 2 apresenta as organizações mais mencionadas pelos grupos de pesquisa no DGP de 2014. No eixo horizontal estão as siglas das organizações e no eixo vertical o número de vezes que foi mencionado como parceiro pelos grupos de pesquisa.

Destaca-se no gráfico da figura 2, de forma surpreendente, a forte presença de universidades entre as organizações parceiras dos grupos de pesquisa. Dentre as organizações mais citadas, 14 são universidades públicas. Além dessas, também são mencionados, em ordem decrescente de frequência, a EMPRAPA (Empresa Brasileira de Pesquisa Agropecuária) que apareceu com 240 citações, o CNPq (Conselho Nacional de Desenvolvimento Cientifico e Tecnológico), com 179 citações, a FIOCRUZ (Fundação Oswaldo Cruz), com 128 citações, e a CAPES (Coordenação de Aperfeiçoamento de Pessoal de Nível Superior), com 103 citações. Isso informa que grande parte das organizações que se relacionam com os grupos de pesquisa interativos das universidades brasileiras são outras universidades públicas ou instituições do setor público.

Precisamos ainda saber: quantas empresas são as parcerias dos grupos, no total e

Econômica-Niterói, v. 20, n. 2, p. 33-58. Dezembro, 2018 
Tabela 7 - Distribuição dos grupos de pesquisa e grupos de pesquisa interativos pela natureza juridica da universidade e região

\begin{tabular}{lcccccccc}
\hline Região & \multicolumn{3}{c}{ Universidades Públicas } & \multicolumn{4}{c}{ Universidades Privadas } \\
\cline { 2 - 9 } & $\begin{array}{c}\text { Total de } \\
\text { Grupos }\end{array}$ & $\begin{array}{c}\text { Partic. } \\
(\%)\end{array}$ & $\begin{array}{c}\text { Total de } \\
\text { Grupos } \\
\text { Interativos }\end{array}$ & $\begin{array}{c}\text { GI } \\
(\%)\end{array}$ & $\begin{array}{c}\text { Total de } \\
\text { Grupos }\end{array}$ & $\begin{array}{c}\text { Partic. } \\
(\%)\end{array}$ & $\begin{array}{c}\text { Total de } \\
\text { Grupos } \\
\text { Interativos }\end{array}$ & $\begin{array}{c}\text { GI } \\
(\%)\end{array}$ \\
\hline Centro-Oeste & 571 & 9 & 64 & 11 & 79 & 7 & 5 & 6 \\
Nordeste & 1264 & 19 & 127 & 10 & 61 & 5 & 8 & 13 \\
Norte & 659 & 10 & 55 & 8 & 31 & 2 & 2 & 6 \\
Sudeste & 2510 & 38 & 238 & 9 & 482 & 45 & 43 & 9 \\
Sul & 1685 & 25 & 126 & 7 & 420 & 39 & 40 & 10 \\
\hline Total Geral & 6689 & 100 & 610 & - & 1073 & 100 & 98 & - \\
\hline
\end{tabular}

Fonte: Elaborado pelos autores com base no Diretório dos Grupos de Pesquisa (DGP) do CNPq, Censo 2014.

por universidade pública e privada. O ideal é termos o gráfico 1 desdobrado em mais 2: citações das universidades públicas e outro com as universidades privadas.

Por fim, foram observadas as dimensões de variáveis anteriormente mencionadas, destacando as diferenças entre universidades (tabela 9).

Existe uma grande presença de grupos pertencentes a universidades da rede pública, são $86 \%$ do total de grupos da amostra selecionada, $86 \%$ do total de grupos interativos e $87 \%$ do total de relacionamentos. O número elevado de interações entre grupos pertencentes a universidades públicas e instituições parceiras acompanha o número elevado de universidades e de grupos registrados na rede pública.

Por outro lado, se avaliarmos a proporção dos grupos interativos com o total de grupos dos dois diferentes tipos de universidade, observa-se o mesmo resultado de 9\%. Dessa forma, concluímos que dentro da amostra, apesar da forte presença do setor publico, o grau de interação é semelhante. As universidades públicas possuem $87 \%$ do total de pesquisadores, porém, possuem um menor número de linhas de pesquisa 79\%. O grupo de universidades privadas conta com $89 \%$ das linhas de pesquisa, demonstrando uma pesquisa mais diversificada.

Embora o setor público mereça destaque, analisando proporcionalmente, as universidades privadas se destacam no número de grupos interativos em relação ao total de relacionamentos (34\% contra $31 \%$ das universidades públicas) e também no número de instituições parceiras (34\% contra 32\%) das universidades públicas. A seguir, analisam-se as variáveis de estrutura de recursos humanos.

As universidades públicas possuem um maior número de grupos com e sem interação registrada no diretório. Ao fim, isso leva a um maior número de registro de recursos 
Tabela 8 - Número de grupos de pesquisa interativos por unidade da Federação

\begin{tabular}{|c|c|c|c|c|}
\hline Unidade da Federação & $\begin{array}{c}\text { Total de } \\
\text { de Grupos } \\
\text { da Amostra } \\
\text { Selecionada }\end{array}$ & $\begin{array}{c}\text { Total de } \\
\text { de Grupos } \\
\text { Interativos }\end{array}$ & $\begin{array}{c}\text { Grupos } \\
\text { Interativos de } \\
\text { Universidades } \\
\text { Públicas }\end{array}$ & $\begin{array}{c}\text { Grupos } \\
\text { Interativos de } \\
\text { Universidades } \\
\text { Privadas }\end{array}$ \\
\hline São Paulo & 1448 & 130 & 106 & 24 \\
\hline Paraná & 830 & 67 & 64 & 3 \\
\hline Minas Gerais & 829 & 88 & 83 & 5 \\
\hline Rio Grande do Sul & 777 & 59 & 31 & 28 \\
\hline Rio de Janeiro & 608 & 54 & 40 & 14 \\
\hline Pará & 537 & 44 & 42 & 2 \\
\hline Santa Catarina & 498 & 40 & 31 & 9 \\
\hline Bahia & 458 & 50 & 45 & 5 \\
\hline Ceará & 209 & 19 & 17 & 2 \\
\hline Paraíba & 184 & 21 & 21 & 0 \\
\hline Distrito Federal & 171 & 15 & 13 & 2 \\
\hline Mato Grosso do Sul & 154 & 18 & 17 & 1 \\
\hline Rio Grande do Norte & 136 & 9 & 9 & 0 \\
\hline Mato Grosso & 133 & 14 & 11 & 3 \\
\hline Espírito Santo & 107 & 9 & 9 & 0 \\
\hline Pernambuco & 96 & 9 & 9 & 0 \\
\hline Amazonas & 78 & 7 & 7 & 0 \\
\hline Sergipe & 69 & 6 & 5 & 1 \\
\hline Maranhão & 66 & 6 & 6 & 0 \\
\hline Alagoas & 65 & 6 & 6 & 0 \\
\hline Piauí & 42 & 9 & 9 & 0 \\
\hline Roraima & 22 & 0 & 0 & 0 \\
\hline Amapá & 18 & 1 & 1 & 0 \\
\hline Rondônia & 17 & 2 & 2 & 0 \\
\hline Acre & 13 & 1 & 1 & 0 \\
\hline Tocantins & 5 & 2 & 2 & 0 \\
\hline Total & 7762 & 708 & 610 & 98 \\
\hline
\end{tabular}

Fonte: Elaborado pelos autores com base em Diretório dos Grupos de Pesquisa (DGP) do CNPq, Censo 2014. 
Tabela 9 - Diferenças de estrutura entre universidades públicas e privadas no Brasil

\begin{tabular}{lcccccc}
\hline & $\begin{array}{c}\text { Total de } \\
\text { Grupos }\end{array}$ & $\begin{array}{c}\text { Total de } \\
\text { Grupos } \\
\text { Interativos }\end{array}$ & $\begin{array}{c}\text { Total de } \\
\text { Interações }\end{array}$ & $\begin{array}{c}\text { Total de } \\
\text { Instituições } \\
\text { Parc. }\end{array}$ & $\begin{array}{c}\text { Pesquisa- } \\
\text { dores }\end{array}$ & $\begin{array}{c}\text { Linhas de } \\
\text { Pesquisa }\end{array}$ \\
\hline $\begin{array}{l}\text { Total da } \\
\text { Amostra }\end{array}$ & 7762 & 708 & 2200 & 2177 & 22120 & 10550 \\
$\begin{array}{l}\text { Universidades } \\
\begin{array}{l}\text { Públicas } \\
\text { Universidades }\end{array}\end{array}$ & 6689 & 610 & 1913 & 1890 & 19294 & 8317 \\
Privadas & 1073 & 98 & 287 & 287 & 2826 & 9361 \\
\hline
\end{tabular}

Fonte: Elaborado pelos autores com base em Diretório dos Grupos de Pesquisa (DGP) do CNPq, Censo 2014.

Tabela 10 - Diferenças de estrutura de recursos humanos entre universidades públicas e privadas brasileiras

\begin{tabular}{lcccc}
\hline & $\begin{array}{c}\text { Pesquisadores } \\
\text { Doutores }\end{array}$ & $\begin{array}{c}\text { Pesquisadores } \\
\text { Mestres }\end{array}$ & $\begin{array}{c}\text { Pesquisadores } \\
\text { Especialistas }\end{array}$ & $\begin{array}{c}\text { Pesquisadores } \\
\text { Graduandos }\end{array}$ \\
\hline Total da Amostra & 17087 & 4129 & 592 & 404 \\
Universidades Públicas & 15186 & 3366 & 498 & 371 \\
Universidades Privadas & 1901 & 763 & 94 & 33 \\
\hline
\end{tabular}

Fonte: Elaborado pelos autores com base em Diretório dos Grupos de Pesquisa (DGP) do CNPq, Censo 2014. 
Figura 2 - Exemplo de cálculo das variáveis para universidades e grupos de pesquisa

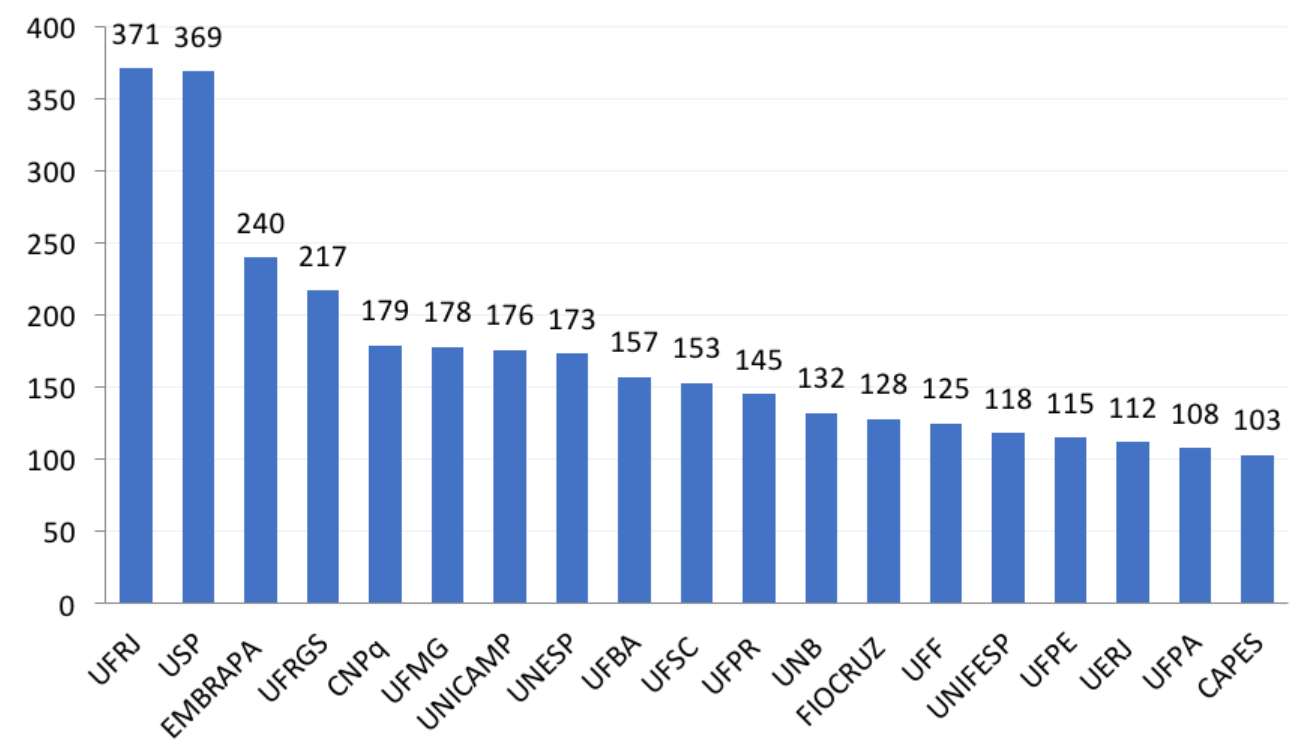

Fonte: Elaborado pelos autores.

humanos. Se observarmos por titulação, o número de pesquisadores doutores é de $89 \%$ contra $11 \%$ das universidades privadas. O percentual de pesquisadores com mestrado é de $82 \%$ nas universidades públicas e $18 \%$ nas privadas. A maior diferença nos percentuais fica no percentual de pesquisadores graduandos, com $92 \%$ nas universidades públicas e $8 \%$ nas universidades privadas. Os pesquisadores Especialistas também apresentam diferenças $84 \%$ das instituições públicas contra $16 \%$ das privadas.

A seguir, a Tabela 11 apresenta os dados da dimensão financeira.

Tabela 11 - Dados financeiros da amostra selecionada de universidades públicas e privadas brasileiras, 2014

\begin{tabular}{lccccccc}
\hline $\begin{array}{l}\text { Universi } \\
\text {-dades }\end{array}$ & $\begin{array}{c}\text { Receita } \\
\text { Própria (R\$) }\end{array}$ & $\%$ & $\begin{array}{c}\text { Investim. } \\
(\mathrm{R} \$)\end{array}$ & $\begin{array}{c}\text { \%Investim. } \\
\text { em Pesquisa }\end{array}$ & $\%$ & $\begin{array}{c}\text { Despesas } \\
\text { Docentes (R } \$)\end{array}$ & $\%$ \\
\hline Públicas & 5144658561 & 18 & 2820420064 & 66707374955 & 64 & 20021483409 & 66 \\
Privadas & 21968584804 & 81 & 1451262152 & 33389827649 & 35 & 10155095306 & 33 \\
\hline Total & 27083243366 & 100 & 4271682217 & 1001097202604 & 100 & 30176578715 & 100 \\
\hline
\end{tabular}

Fonte: Elaborado pelos autores com base em Diretório dos Grupos de Pesquisa (DGP) do CNPq, Censo 2014.

As variáveis financeiras também apresentam diferenças, as Universidades Públicas registram $66 \%$ do total de Investimentos da amostra analisada. Se observarmos os inves-

Econômica-Niterói, v. 20, n. 2, p. 33-58. Dezembro, 2018 
timentos registrados em pesquisa, o percentual é de $64 \%$ contra $34 \%$ das universidades privadas. Se tratando de receita própria, como esperado, as universidades privadas, possuem um total superior, com $81 \%$ do total contra $19 \%$ nas universidades públicas.

As despesas docentes também são distantes, porém com superioridade para as Universidades Públicas, que realizam $66 \%$ do total dos valores da amostra destinados a este fim, enquanto as universidades privadas utilizam $34 \%$. Ao analisarmos o percentual de investimento em pesquisa contra o total de investimento nos dois diferentes grupos de instituições temos o seguinte resultado: $25 \%$ para universidades públicas e $27 \%$ para universidades privadas. Frente a esta analise, podemos observar um esforço maior do setor privado em pesquisa, embora em números absolutos o resultado de interação do grupo de universidades publica seja mais efetivo, como podemos verificar na tabela 12.

Tabela 12 - Grau e Diversidade da interação com organizações entre universidades públicas e privadas brasileiras

\begin{tabular}{lcc}
\hline & $\begin{array}{c}\text { Grau de interação } \\
\text { da instituição (\%) }\end{array}$ & $\begin{array}{c}\text { Media da Diversidade de } \\
\text { Interação dos Grupos (\%) }\end{array}$ \\
\hline Total da Amostra & 0,091 & 0,2207 \\
Universidades Públicas & 0,091 & 0,3329 \\
Universidades Privadas & 0,091 & 0,0947 \\
\hline
\end{tabular}

Fonte: Elaborado pelos autores com base em Diretório dos Grupos de Pesquisa (DGP) do CNPq, Censo 2014.

Os indicadores estão calculados para os grupos de pesquisa, então o cálculo para os grupos de universidades foi realizado utilizando a média ponderada entre os grupos para obter o resultado de cada instituição e, posteriormente, a média para cada um dos grupos distintos (público x privado). O Grau de interação é composto pela proporção entre o total de grupos com relacionamento da instituição dividido pelo total de grupos registrados, apresenta média semelhante entre os diferentes grupos de IES com 0,091 para ambas e o mesmo resultado para média total. Demonstrando que ambos os tipos de universidades são interativos quase que na mesma proporção. A diversidade de interação é calculada pela proporção do número de relacionamentos do grupo dividido pelo total dos 14 tipos diferentes registrados no CNPq. Para calcular os resultados dos diferentes grupos de universidades, utilizou-se a média dos resultados dos grupos. Os resultados obtidos foram 0,3329 para IES públicas e 0,0947 para IES privadas. Demonstrando que as universidades públicas possuem maior diversidade nas interações registradas. Possivelmente essa diferença se apresenta em função do número elevado de grupos com relacionamento registrado nas universidades públicas As variáveis de produção dos grupos, diferenciando universidades públicas e privadas pode ser observado na Tabela 13 a seguir.

As variáveis de resultado dos grupos de pesquisa apresentam diferenças significativas. Grupos de pesquisa de universidades públicas possuem maior número de produção 
Tabela 13 - Diferenças de produção entre universidades públicas e privadas brasileiras.

\begin{tabular}{lccc}
\hline & Publicações & Produção Técnica & Orientações \\
\hline Total da Amostra & 31620 & 12035 & 17527 \\
Universidades Públicas & 27678 & 10226 & 15387 \\
Universidades Privadas & 3942 & 1809 & 2140 \\
\hline
\end{tabular}

Fonte: Elaborado pelos autores com base em Diretório dos Grupos de Pesquisa (DGP) do CNPq, Censo 2014.

nos três diferentes tipos, sendo $88 \%$ do total de publicações, $85 \%$ no total de produção técnica e $88 \%$ no total de orientações. As universidades privadas possuem resultados modestos se avaliarmos apenas o número total, porém ao observamos a proporção das publicações levando em consideração o número de grupos de cada tipo de instituição observamos um resultado um pouco mais equilibrado com as seguintes proporções: No total de publicações as universidades apresentam o total de 2,20\% públicas contra 2,49\% das universidades privadas. Um resultado semelhante para a razão grupos interativos e publicações. No total de produção técnica essa diferença aumenta para 6\% nas universidades públicas e $5 \%$ nas universidades privadas e no total de orientações $4 \%$ nas instituições públicas contra $5 \%$ nas privadas.

Analisando a proporção do número de grupos de cada universidade sobre os resultados, as instituições privadas possuem número superior nas publicações e orientações. E um resultado bem próximo no total de produção técnica.

Evidenciando a superioridade da rede pública na amostra selecionada, o Quadro da tabela 14 a seguir mostra o percentual de cada uma das variáveis utilizadas nesta pesquisa para cada conjunto de universidades.

As universidades públicas possuem maior percentual na maioria das variáveis. Embora a superioridade do grupo das públicas seja confirmada no Quadro 2, a interação se mostra semelhante no cálculo do grau de interação. Especificamente, o conjunto de grupos pertencentes às universidades privadas possuem melhor resultado na receita própria obtida e no número de linhas de pesquisa de registro dos grupos de pesquisa.

\section{Considerações Finais}

Este trabalho aponta as características da interação de grupos de pesquisa cadastrados no DGP/2014 pertencentes a universidades públicas ou privadas.

Verificou-se a existência de 7.762 grupos de pesquisas registrados no DGP/2014 com 708 grupos interativos. São 6.689 grupos de pesquisa registrados em universidades púbicas, sendo destes, 610 grupos interativos. E no conjunto de universidades privadas, 1.073

Econômica-Niterói, v. 20, n. 2, p. 33-58. Dezembro, 2018 
Tabela 14 - Resultados percentuais das variáveis utilizadas na pesquisa

\begin{tabular}{|c|c|c|c|}
\hline & Variáveis & $\begin{array}{l}\% \text { Universidades } \\
\text { Públicas }\end{array}$ & $\begin{array}{c}\% \text { Universidades } \\
\text { Privadas }\end{array}$ \\
\hline \multicolumn{4}{|c|}{1 - Variáveis de Estrutura } \\
\hline QTDGP & $\begin{array}{l}\text { Quantidade de grupos de } \\
\text { pesquisa }\end{array}$ & 86 & 14 \\
\hline QTDPREL & $\begin{array}{l}\text { Quantidade de grupos de } \\
\text { pesquisa interativos }\end{array}$ & 86 & 14 \\
\hline TOTALREL & Total de relacionamentos & 86 & 14 \\
\hline QTDEMPR & $\begin{array}{l}\text { Quantidade de instituições } \\
\text { parceiras }\end{array}$ & 86 & 14 \\
\hline TOTPESQ & $\begin{array}{l}\text { Total de pesquisadores } \\
\text { dos grupos interativos }\end{array}$ & 87 & 13 \\
\hline TOTEST & $\begin{array}{l}\text { Total de Estudantes dos } \\
\text { grupos Interativos }\end{array}$ & 88 & 12 \\
\hline LPESQ & Linhas de Pesquisa & 11 & 89 \\
\hline \multicolumn{4}{|c|}{2 - Estruturas de Recursos Humanos } \\
\hline PESDOUT & Pesquisadores Doutores & 88 & 12 \\
\hline PESQMEST & Pesquisadores Mestres & 81 & 19 \\
\hline PESQESPEC & Pesquisadores Especialistas & 85 & 15 \\
\hline PESQGRAD & Pesquisadores Graduandos & 92 & 8 \\
\hline \multicolumn{4}{|c|}{3 - Interação } \\
\hline GII & $\begin{array}{l}\text { Grau de interação da } \\
\text { instituição }\end{array}$ & 9 & 9 \\
\hline DII & $\begin{array}{l}\text { Diversidade de interação } \\
\text { da Instituição }\end{array}$ & 33 & 9 \\
\hline \multicolumn{4}{|c|}{4 - Investimento e Despesas Institucionais } \\
\hline RECPROPRIA & Receita Própria & 18 & 81 \\
\hline VALDESPINV & Investimento Total & 66 & 33 \\
\hline VALDESPEQU & Investimento em pesquisa & 64 & 35 \\
\hline VALDESPDOC & Valor das despesas docentes & 66 & 33 \\
\hline \multicolumn{4}{|c|}{5 - Resultados dos Grupos de Pesquisa } \\
\hline TOTPUBLIC & Total de Publicações & 88 & 12 \\
\hline TOTPRODTEC & Total de Produção Técnica & 85 & 15 \\
\hline TOTORIENT & Total de Orientações & 88 & 12 \\
\hline
\end{tabular}

Fonte: Elaborado pelos autores com base em Diretório dos Grupos de Pesquisa (DGP) do CNPq, Censo 2014. 
grupos, sendo 98 grupos interativos. A maior parte dos registros dos grupos ocorre na área de pesquisa de Ciências da Saúde com 20\% dos registros, acompanhado por $17 \%$ nas Ciências Humanas e outros 16\% nas Ciências Sociais Aplicadas. Ao analisar os dados dos grupos interativos, a área da Ciência da Saúde e a área de ciências Humanas apresentam os maiores percentuais com 20\% dos registros, seguido por Ciências Sociais Aplicadas com $15 \%$ e Ciências Exatas e da Terra com 11\%. Do total de grupos de pesquisa registrados nas universidades da amostra, apenas $9 \%$ possui algum tipo de relacionamento.

Observou-se, uma grande presença de grupos interativos pertencentes a universidades públicas, $86 \%$ do total da amostra, conduzindo a resultados superiores deste tipo de universidade também nas demais variáveis pesquisadas: $86 \%$ na quantidade de grupos de pesquisa, $87 \%$ do total de relacionamentos registrados, $87 \%$, do número de pesquisadores registrados, $33 \%$ de diversidade na interação e $66 \%$ do total de despesas docentes. Assim, pôde-se observar que as universidades públicas possuem um número maior de interações, e uma estrutura docente mais qualificada, o que segundo a literatura, pode ter como um dos fatores explicativos as raízes históricas mais antigas deste tipo de universidade no contexto brasileiro.

Contudo, vale destacar que os resultados apontaram para um grau de interação dos grupos de pesquisa semelhante, ou seja, analisando proporcionalmente o número de grupos registrados e o número de grupos interativos dos dois conjuntos de universidades, não há diferença. Ou seja, Apesar das universidades públicas possuírem elevado número de grupos registrados, e possuírem melhor resultado nas variáveis analisadas, a interação se mostra relativamente igual quando verificada desta forma.

As variáveis de resultado registradas pelos grupos reforçam este pensamento. Ao observar as universidades privadas, os resultados são inferiores em número absoluto. Todavia, ao considerar a proporção do resultado contemplando o número total de grupos dos dois diferentes tipos de universidades as diferenças percebidas são minimizadas, mostrando que a intensidade de produção dos grupos de universidades privadas também deve ser considerada em futuros estudos. Geograficamente, as regiões Sul e Sudeste concentram a maior parte dos relacionamentos registrados, enquanto as demais regiões se dividem em pequenos percentuais. Observando o Grau de interação as regiões destacadas apresentam mudanças. A rede pública aparece nas regiões Centro Oeste e Nordeste, enquanto que as universidades privadas possuem maiores registros na região Sul e Nordeste.

O tipo de relacionamento "Pesquisa científica sem considerações de uso imediato dos resultados" é o mais registrado pelos grupos de universidades de ambas as naturezas jurídicas, com $81 \%$ dos registros pertencentes a universidades privadas e $96 \%$ nas universidades públicas. Este resultado reflete a necessidade da demanda das empresas que buscam relacionamento com os grupos de pesquisa no Brasil, seu grande percentual de registro nos dois conjuntos de universidades permite observar que o caráter da pesquisa é o mesmo, indiferente da natureza jurídica da universidade com que a empresa realiza a interação. 
Considerando a dimensão financeira, o conjunto de universidades públicas apresentam números superiores, entretanto, as universidades privadas possuem resultado elevado no total da receita própria da amostra $(81 \%)$ e possuem menores despesas com o quadro docente (33\%). Por outro lado, são responsáveis por $35 \%$ de investimento em pesquisa e $33 \%$ de investimento total.

Nos indicadores de resultado, observando os números absolutos existe uma superioridade do conjunto de grupos de universidades públicas, destacando-se com $88 \%$ do total de publicações, $85 \%$ da produção técnica e $88 \%$ do total de orientações.

A diferença observada entre o número de grupos dos dois conjuntos de universidades também nos permite entender o motivo do elevado número de estudos envolvendo universidades públicas na literatura brasileira. As universidades privadas possuem uma intensidade de interação e de pesquisa semelhante à rede pública, mas por vezes subdimensionada em virtude do número absoluto de grupos registrados ser bastante inferior.

Em um país que encontra cada vez mais dificuldades em absorver a demanda por pesquisa e ensino superior através do investimento público, onde as universidades são parte importante da infraestrutura cientifica, as diferenças apresentadas nesta pesquisa possuem importância no sentido de auxiliar a discussão a respeito da pesquisa pública e privada e seus impactos, bem como no olhar que deve-se ter para as universidades quando de estudos a respeito de Sistemas Nacionais de Inovação e sua importância para o desenvolvimento dos países.

\begin{abstract}
Universities are recognized as producers and disseminators of scientific and technological knowledge and can influence the generation and diffusion of innovations. For the Brazilian reality, the literature indicates a relatively low volume of interactions between universities and organizations, considering the immaturity of the country's National Innovation System (SNI). In addition, it is understood that public universities, as opposed to private universities, are institutions with a greater presence in the interaction scenario, due to the historical trajectory of constitution and development in the longer term in the Brazilian education and science and technology system, as well as by the fact that they enjoy more resources, both human and financial, for the generation and transmission of knowledge. In this context, it is understood to be relevant to understand the interactions established according to the legal nature of the universities. An exploratory study was carried out using secondary data from the CNPq Research Group Directory (CNPq) and the INEP census of higher education for the year 2014. The results indicated that public universities have a greater number of registers of research groups, interactive research groups, doctors, masters and other resources in relation to private universities. Its main knowledge areas of the interactive groups are health, human, exact and earth sciences. The interactive groups of private universities are mainly in the areas of applied social sciences, engineering, and biological sciences. Another interesting and surprising result was the calculation of the degree of interaction that indicated to have been similar for the two groups of universities analyzed. This requires a more accurate and inclusive view of the role of private universities in the scenario of university-organization interac-
\end{abstract}


tion in Brazil.

Keywords: Interaction University and Organizations, Public and Private Universities, Interactive Research Groups, National System of Brazilian Innovation.

JEL: O33

\section{Referências bibliográficas}

ALBUQUERQUE, E. D. M. Sistema nacional de inovação no Brasil: uma análise introdutória a partir de dados disponíveis sobre a ciência e a tecnologia. Revista de Economia Política, v. 16, n. 3, p. 56-72, 1996. Disponível em: <http://www.scielo.br/ scielo.php?script=scil_nlinks\&ref=000130\&pid=S1517-9702201200040000800002\& $\operatorname{lng}=\mathrm{pt}>$.

BRUNDENIUS, C.; LUNDVALL, B.-; SUTZ, J. The Role of Universities in Innovation Systems in Developing Countries: Developmental University Systems - Empirical, Analytical and Normative Perspectives. In: LUNDVALL, B.- et al. (Ed.). Handbook of Innovation Systems and Developing Countries: building domestic capabilities in a global setting. [S.1.]: Edward Elgar Publishing, 2009. cap. 11. ISBN 9781847206091.

CHAIMOVICH, H. Por uma relação mutuamente proveitosa entre universidade de pesquisa e empresas. Revista de Administração, v. 34, n. 4, p. 18-22, 1999. Disponível em: <http://www.spell.org.br/documentos/ver/18141/ por-uma-relacao-mutuamente-proveitosa-entre-universidade-de-pesquisa-e-empresas/i/ pt-br>.

DURHAM, E. R. O ensino Superior no Brasil: Público e Privado. Núcleo de Pesquisa sobre Ensino Superior da Universidade de São Paulo - NUPES. In: Seminário sobre Educação no Brasil. [s.n.], 2003. Disponível em: <http: //nupps.usp.br/downloads/docs/dt0303.pdf>.

FREEMAN, C. The 'National System of Innovation' in historical perspective. Cambridge Journal of Economics, Oxford University Press, v. 19, n. 1, p. 5-24, 2 1995. ISSN 1464-3545. Disponível em: <https://academic.oup.com/cje/article/19/1/5/1708372/ The-National-System-of-Innovation-in-historical $>$.

LUNDVALL, B.-A. National systems of innovation : toward a theory of innovation and interactive learning. Anthem, 1992. 388 p. ISBN 9780857286741. Disponível em: $<\mathrm{https}: / / \mathrm{www}$.jstor.org/stable/j.ctt1gxp7cs>.

Econômica-Niterói, v. 20, n. 2, p. 33-58. Dezembro, 2018 
MEYER-KRAHMER, F.; SCHMOCH, U. Science-based technologies: university-industry interactions in four fields. Research Policy, v. 27, n. 8, p. 835-851, 12 1998. ISSN 00487333. Disponível em: <http://linkinghub.elsevier.com/retrieve/pii/S0048733398000948>.

NELSON, R. R. National innovation systems : a comparative analysis. [S.1.]: Oxford University Press, 1993. 541 p. ISBN 9780195076172.

PINHO, M. A Visão das empresas sobre as relações entre Universidade e empresa no Brasil: uma análise baseada nas categorias de intensidade tecnológica. Revista de Economia, v. 37, n. Especial, p. 279-306, 2011.

PUFFAL, D. P.; RUFFONI, J.; SCHAEFFER, P. Características da interação universidade-empresa no Brasil: motivações e resultados sob a ótica dos envolvidos. Gestão Contemporânea, n. especial, 2012.

RAPINI, M. S. Interação universidade-empresa no Brasil: evidências do Diretório dos Grupos de Pesquisa do CNPq. Estudos Econômicos (São Paulo), Instituto de Pesquisas Econômicas da FEA-USP, v. 37, n. 1, p. 211-233, 3 2007. Disponível em: <http: //www.scielo.br/scielo.php?script=scil_arttext\&pid=S0101-41612007000100008\&lng= $\mathrm{pt} \& \mathrm{t} \operatorname{lng}=\mathrm{pt}>$.

RAPINI, M. S.; RIGHI, H. M. Interação Universidade-Empresa no Brasil em 2002 e 2004: Uma aproximação a partir dos Grupos de pesquisa do CNPQ. Revista Economia, v. 8, n. 2, p. 248-268, 2007.

ROSA, A. C. D.; RUFFONI, J. Capacidade Absortiva de Firmas que utilizam Universidades como Fonte de Conhecimento Externo. In: $41^{\circ}$ Encontro Nacional de Economia (Ed.). $41^{\circ}$ Encontro Nacional de Economia. [S.1.]: Anpec, 2013.

SCHAEFFER, P. R.; RUFFONI, J.; PUFFAL, D. Razões, benefícios e dificuldades da interação universidade-empresa. Revista Brasileira de Inovação, v. 14, n. 1, p. 105, 2 2015. Disponível em: <https://periodicos.sbu.unicamp.br/ojs/index.php/rbi/article/view/ $8649091>$.

SCHARTZMAN, S. Formação da Comunidade Cientifica no Brasil. São Paulo: Companhia Editora Nacional e Finep, 1979. 481 p.

SCHARTZMAN, S. A democracia e o futuro das universidade, Simon Schwartzman. Presença - Revista de Política e Cultura, n. 7, 1986. Disponível em: <http: //www.schwartzman.org.br/simon/presen.htm>.

SCHUMPETER, J. A. Capitalismo, socialismo e democracia. xvi. Rio de Janeiro: Fundo de Cultura, 1961. $512 \mathrm{p}$. 
SCHUMPETER, J. A. A Teoria do Desenvolvimento Econômico. São Paulo:: Abril Cultural, 1982.

SGUISSARDI, V. Universidade pública estatal: entre o público e privado/mercantil. Educação \& Sociedade, v. 26, n. 90, p. 191-222, 4 2005. ISSN 0101-7330.

Disponível em: <http://www.scielo.br/scielo.php?script=scil_arttext\&pid= S0101-73302005000100009\&lng=pt\&tlng=pt $>$.

SILVA, F. R. V. e. Análise da interação universidade-empresa a partir das diferenças em instituições públicas de ensino superior no Brasil. 305 p. Tese (Doutorado) - UFRGS, 2016. Disponível em: <https://lume.ufrgs.br/handle/10183/150513>.

SILVA, R. R. et al. Em Busca da Interação: uma análise do comportamento das interações entre universidades e empresas. In: XVI Congresso Latino-Iberoamericano de Gestão da Tecnologia - ALTEC 2015 (Ed.). XVI Congresso Latino-Iberoamericano de Gestão da Tecnologia - ALTEC 2015. Porto Alegre: ALTEC, 2015.

STAL, E. et al. The evolution of universities' relations with the business sector in Brazil: What national publications between 1980 and 2012 reveal. Revista de Administração, Departamento de Administração da Faculdade de Economia, Administração e Contabilidade da Universidade de São Paulo, v. 51, n. 1, p. 072-086, 2016. Disponível em: <http://200.232.30.99/busca/artigo.asp?numl_artigo=1676>.

SUZIGAN, W.; ALBUQUERQUE, E. d. M. e. Interações de Universidades e institutos de pesquisa com empresas no Brasil. 2006.

SUZIGAN, W.; ALBUQUERQUE, E. d. M. e. A interação entre universidades e empresas em perspectiva histórica no Brasil. 2008. Disponível em: <https://cedeplar.ufmg.br/en/publicacoes/textos-para-discussao/textos/2008/ 592-329-a-interacao-entre-universidades-e-empresas-em-perspectiva-historica-no-brasil>.

Econômica-Niterói, v. 20, n. 2, p. 33-58. Dezembro, 2018 